\title{
$\nabla$ \\ Diode Laser 1470 nm versus Diode Laser 980 nm for Treatment of Primary Axillary Hyperhidrosis
}

\section{IJCRR}

Section: Healthcare

ISI Impact Factor

(2020-21): 1.899

IC Value (2020): 91.47

$\operatorname{SJIF}(2020)=7.893$

(c) (i) (3)

Copyright@IJCRR

\section{Diaa Aldin Sayed Ibrahim¹, Mahmoud S. Elbasiouny², Tarek F. Elwakil², Nevien Ahmed Samy², Maha Rafie AboEitta²}

'Medicine and Clinical Studies Research Institute, National Research Center, Cairo University, Egypt; ${ }^{2}$ Department of Medical Applications of Lasers, National Institute of Laser Enhanced Science, Cairo University, Egypt.

\section{ABSTRACT}

Introduction: Primary axillary hyperhidrosis $(\mathrm{PAH})$ is a distressing condition that can pose significant social embarrassment. Different treatments such as topical agents, systemic agentsand botulinum toxin are only temporarily effective. Thus, there is a need for evidence-based management. The following study describes a new technique for the management of (PAH). We study the efficacy and safety of the subdermal application of two different diode lasers, at $980 \mathrm{~nm}$ or $1470 \mathrm{~nm}$, for ablation of the sweat glands versus intradermal Botulinum toxin A (BTA).

Patients and Methods: This parallel-randomized controlled pilot study was conducted upon 90 PAH patients from December-2019 to July-2021 at the National Institute of Laser Enhanced Sciences (NILES), Egypt; the diode laser $980 \mathrm{~nm}$ group (30 cases), the diode laser $1470 \mathrm{~nm}$ group (30 cases) and the BTA group (30 cases).

Results: The three groups were comparable regarding age, BMI, and gender. The laser groups had higher reductions of HDSS score than the BTA group at 1-month $1.37 \pm 0.49$ and $1.20 \pm 0.41$ versus $2.03 \pm 0.67$ for the laser 980,1470 and the BTA groups, respectively ( $p$ values $<0.001$ ) and at 6 -months $(1.37 \pm 0.48$ and $1.20 \pm 0.41$ versus $2.20 \pm 0.55 ; p$-value $<0.001)$. At 1 -month and 6 -months, the laser groups had higher reduction of mean moisture percentages than the BTA group at 1-month with $49.34 \pm 5.88 \%$ and $48.93 \pm 6.62 \%$ versus $55.89 \pm 9.20 \%$, respectively (p-values $0.003 \& 0.001$ ) and at 6 -months $49.42 \pm 6.32 \%$ and $48.90 \pm 6.79 \%$ versus $56.21 \pm 7.98 \%$, respectively ( $p$-values $0.001 \&<0.001$ ).

At 1-month and month-6, there was a higher patient satisfaction in the laser groups than in the BTA group ( $p$-values < 0.05 ). The laser groups demonstrated better GAIS score than the BTA group at both 1-month (p-values $<0.05)$ and 6 -months visits (p-values<0.001).

Conclusion: The study showed that Diode laser $1470 \mathrm{~nm}$ is safer and more effective treatment for PAH than Diode laser 980 $\mathrm{nm}$.

Key Words: Botulinum toxin A, Diode 980 nm laser, Diode $1470 \mathrm{~nm}$ laser, Primary axillary hyperhidrosis, Quality of life, Patients' satisfaction

\section{INTRODUCTION}

The armamentarium for the management of primary axillary hyperhidrosis (PAH) is still in great need of evidence-based management. The current management of PAH includes topical therapies like antiperspirants and aluminum chloride. Nonetheless, they usually have temporary effects with a variety of side effects such as itching \&burning sensations or skin rashes ${ }^{1,2,3}$. Another alternative that is being used as standard therapy for $(\mathrm{PAH})$ is the intradermal injection of Botulinum toxin type A (BTA), which efficiently suppresses sweat secretion at the level of presynaptic cholinergic neurons. ${ }^{1,4,5,6-10}$

\section{Corresponding Author:}

Diaa Aldin Sayed, Medicine and Clinical Studies Research Institute,National Research Centre (NRC), Cairo University, Cairo, Egypt. Phone: +2 01222187248; Email: dr.diaaaldin1978@gmail.com

ISSN: 2231-2196 (Print) ISSN: 0975-5241 (Online)

Received: $09.12 .2021 \quad$ Revised: 24.12 .2021

Accepted: 19.01 .2022 
this approach offers extremely good long-term results with a low amount of temporary adverse effects. ${ }^{17}$

Aydin and colleagues recently described an increase in hyperhidrosis activity after axillary hair removal using a 1064$\mathrm{nm} \mathrm{Nd}$ : YAG laser in 38 patients. Sweat production was significantly higher than before hair removal as evaluated using analogous scales and the iodine starch test. The authors supposed that a sympathetic skin response after laser stimulation could be induced through thermal heat, which functions as a stimulus for nerve fibers that innervate eccrine glands. This means the use of non-contact laser through the skin as during hair removal may have no effect on sweat production hence the thinking of interstitial subdermal heating. ${ }^{18}$

In addition, interstitial laser technology has been employed successfully in the management of PAH where a cannula was used to introduce the fiber into the area under treatment. Then, it is moved back and forth over the surface under treatment. ${ }^{19-21}$

The effectiveness and safety of this method have been studied extensively in clinical trials. Maletic and Goldman performed two of them, which provided the most precise data on the procedure employed and the results achieved..$^{11,22}$ In these two investigations, the authors employed short-pulse $\mathrm{Nd}$ :YAG lasers that were originally designed for laser liposuction. Subcutaneous (SC) infiltration with Klein's solution is used to provide tumescent anesthesia. The energy levels utilized are comparable and are determined by the treatment area. The operation took half an hour, then for the next two days, dressings and bandages were applied. ${ }^{19}$

Goldman A et al. (2008) intended to show the Nd-YAG laser as a safe and effective technique for PAH treatment in their study. Their research found that treating PAH with a subdermal 1,064-nm Nd-YAG laser resulted in considerable clinical improvement. The mean reduction of the Minor's test visualized area was 72 percent during a follow-up period of one to two years. In 82 percent of the cases, patients were satisfied. ${ }^{11,19}$

Maletic D et al. (2011) investigated the effectiveness and safety of treating PAH with a subdermal $1064 \mathrm{~nm}$ Nd: YAG laser. At 1-3 months after the procedure, their study found a 93 percent reduction in sweating area. The research documented transitory side effects and offered some evidence for the procedure's effectiveness. ${ }^{19,22}$

There are some published studies regarding the effects of different wavelengths, but there are little data comparing their clinical outcomes. A study using lasers with wavelengths of 810, 940-, and 980-nm found that these wavelengths cause steam bubbles to form in blood-filled veins. Veins, on the other hand, showed relatively minor wall damage. The wavelengths 1470 and $1320 \mathrm{~nm}$ are targeted at the water, and energy at both wavelengths is directly delivered to the collagen in the vessel wall. ${ }^{23}$
Therefore, the aim of this current study was to compare the efficacy and safety of two different laser intensities, diode laser $980 \mathrm{~nm}$ or diode laser $1470 \mathrm{~nm}$, versus intradermal Botulinum toxin $\mathrm{A}$ in the management of PAH.

\section{Patients and Methods}

This study was carried out at the National Institute of Laser Enhanced Sciences (NILES) Cairo university outpatient clinics, the study includes 90 patients with PAH from December 2019 to June 2021. The ethical review committee of Cairo University approved the study. The purpose of this study was clearly explained to all patients before their enrollment.

For inclusion in the study, all of the following criteria were to be fulfilled: healthy male or female between $18-64$ years of age suffering from excessive axillary hyperhidrosis, clinically diagnosed as PAH, with the Hyperhidrosis Disease Severity Scale (HDSS)or Malodour score of three or four. ${ }^{24,25}$ Patients understand and accept the obligation to refrain from receiving any other procedures in anatomical areas exhibiting axillary hyperhidrosis for at least three months prior to treatment, and they are able to attend all scheduled follow-up visits after signing an informed consent form.

Exclusion criteria included active bacterial or fungal infection, hidradenitis suppurativa, pregnancy or planned pregnancy or breastfeeding, clinical diagnosis of secondary hyperhidrosis, a history of autoimmune connective tissue disorders, any medical or chronic disease interferes or being contraindicated to the maneuver as significant cardiovascular disease or bleeding disorders, patients who had previous axillary laser hair reduction nor plan to have laser hair reduction while in the study, patients who had previous surgical treatment of the axillae such as axillary gland excision, liposuction, sympathectomy, or subcutaneous curettage, history of sensitivity to Lidocaine (Local Anesthesia) or Epinephrine, and patients who refused to sign the informed consent document and/or refused to comply with all follow-up requirements. All patients in all groups have laboratory assessment in the form of complete blood count (CBC), prothrombin time (PT), international normalized ratio (INR), random blood sugar (RBS), and TSH, T3 \& T4.

\section{Study design}

This three-arm parallel-randomized controlled trial and for allocation of the participants, a computer-generated list of random numbers was used. Patients were randomized into three (group I): treated with subdermal diode laser $980 \mathrm{~nm}$ (group II): treated with subdermal diode laser $1470 \mathrm{~nm}$ (group III): treated with botulinum toxin (Botox) intradermal injection.

\section{Laser irradiation}

Laser irradiation was done on the subdermal side of the skin with a class 4, diode laser $980 \mathrm{~nm}$ (quanta system spa 21058 
Solbiate Olona (VA), Italy) in the CW mode of laser emission. The other laser used was Laser $1470 \mathrm{~nm}\left(\right.$ VELAS $^{\mathrm{TM}}$ by GIGA Laser, Wuhan, China) used also in the same CW mode of emission. The laser light is conveyed through an optical fiber with a diameter of $600 \mu \mathrm{m}$ the power used in the range of 5-7 watts for both types of laser.(As illustrated in Figures 3 and 4$)$.

\section{Lasing procedure}

Before the procedure, room environmental temperature was ensured within $24-26$ Celsius degrees, and skin temperature was measured using a skin infrared thermometer. Physical examination was done to confirm the excessive axillary sweating with a bad foul odor.

The hair-bearing area of the axilla was marked in the supine position The laser fiber optic through a microcannula with a diameter of $2.0 \mathrm{~mm}$ into which an optical fiber of $600 \mu \mathrm{m}$ is inserted after routine tumescent anesthesia, two small punctures were made with an 18-gauge needle at the anterior and distal border of each axilla. The cannula was inserted into the target layer of the subdermal junction. Position and depth of the cannula tip were controlled by transcutaneous guidance with a red aiming beam light. The laser was applied to the tissue in a criss-cross manner (fanning technique). Cold packs were applied to the treated area to prevent heat injury of the skin during the operation. The procedure in one side took 10-12 minutes. Thermal camera was used during lasing procedure and according to its readings the temperature did not exceed 40-45 degree Celsius as illustrated in figure (5.A) and (5.B).

For the BTA group, (BOTOX A 100 units) injecting two units of BOTOX in every point covering the axilla.

\section{Evaluations}

Clinical assessment was performed by the clinical scoring of hyperhidrosis (Hyperhidrosis Disease Severity Scale HDSS) and the Malodour score test to determine the degree of sweating. Scores (3) and (4) in both scorings are considered positive and suffer from excessive axillary hyperhidrosis and Malodor.

Iodine starch test will be done (before and after) the procedure, in which tincture of iodine solution and starch are applied together on both axillae. After ten to fifteen minutes, the color changed from white to blue. A positive iodine starch test indicates the presence of hyperhidrosis. (As illustrated in Figure 1).

The sweating level was determined by (Digital Moisture Monitor for skin), a sweat moisture analyzer that measures the degree of moisture of the skin of axillae. A digital infrared skin thermometer was used to ensure measuring the skin temperature all through the procedure to avoid skin injury by burning.

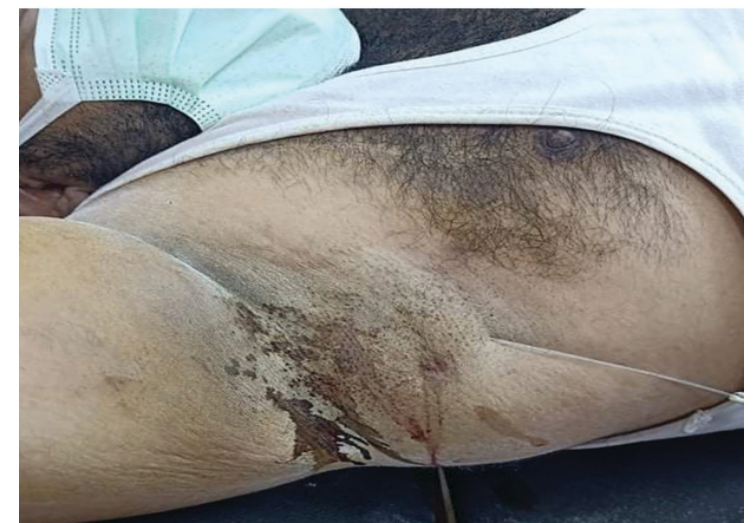

Figure 1: lodine Starch Test Preoperatively.

The degree of patient stratification was also be facilitated by the use of structured questionnaires for the patient's satisfaction and Global Aesthetic Improvement Scale (GAIS) Score. ${ }^{26}$ These ensure consistency and objectivity of approach in the assessment and provide outcome measures that are increasingly recommended for use in clinical practice. The questionnaire responses regarding malodor and sweating were based on subjective data from the patient and their family and friends.

Follow-up visits: All patients were followed-up for six months, including assessment at one month and another at six months.

\section{Outcome measures:}

The primary outcome measure was assessing the efficacy of the three interventions via HDSS at month $1 \& 6$, improvement on the patient satisfaction score (PSS) at month $1 \& 6$, and the Global Aesthetic Improvement Scale (GAIS) Score at month $1 \& 6$. The secondary outcomes were malodor score at month $1 \& 6$, sweating level determined by (Digital Moisture Monitor) at month $1 \& 6$ and quality of life by the DLQI questionnaire at month $1 \& 6$.

\section{Statistical methods}

All statistical tests were conducted at a significance level of $5 \%$ on both sides. A p-value of $<0.05$ is considered statistically significant. SPSS software (Statistical Package for the Social Sciences, version 24.0, SSPS Inc., Chicago, IL, USA) was used. Quantitative parametric data were demonstrated as mean and standard deviation (mean $\pm \mathrm{SD}$ ).

Quantitative non-parametric data were presented as the median and interquartile range (IQR). Qualitative data were presented as numbers and proportions. The Chi-square test was calculated to compare between the three groups for nominal variables. Repeated measures ANOVA tests were used for whether there are any differences between related means. Post-hoc tests were used to detect the differences between groups. 


\section{RESULTS}

The three study groups were comparable as regards to age ( $p$ 0.716), gender ( $p$ 0.95), and BMI ( $\mathrm{p}$ 0.733). The mean age was $38.03 \pm 11.12,37.00 \pm 11.65 \& 35.63 \pm 11.36$ years for the laser 980 group, the laser 1470 group \& the BTA group, respectively. Females constituted $66.67 \%, 66.67 \%$ \& $70 \%$ of cases in the laser 980 group, the laser 1470 group \& the BTA group, respectively. The mean BMI was $29.77 \pm 3.94$, $29.07 \pm 3.21 \& 29.55 \pm 3.36 \mathrm{~m}^{2}$ for the laser 980 group, the laser 1470 group \& the BTA group, respectively.

Table 1: Efficacy, satisfaction and QOL in both groups

\begin{tabular}{|c|c|c|c|c|c|c|c|}
\hline \multirow[b]{2}{*}{ HDSS } & \multicolumn{2}{|c|}{ Diode 980} & \multicolumn{2}{|c|}{ Diode 1470} & \multicolumn{2}{|c|}{ BTA } & \multirow{2}{*}{$\begin{array}{l}\text { ANOVA } \\
\text { p-value }\end{array}$} \\
\hline & Mean & SD & Mean & SD & Mean & SD & \\
\hline Baseline & $3 \cdot 70$ & 0.47 & $3 \cdot 57$ & 0.50 & 3.63 & 0.49 & 0.572 \\
\hline 1-month & 1.37 & 0.49 & 1.20 & 0.41 & 2.03 & 0.67 & $<0.001$ \\
\hline 6-months & 1.33 & 0.48 & 1.20 & 0.41 & 2.20 & 0.55 & $<0.001$ \\
\hline \multicolumn{8}{|c|}{ Malodour score } \\
\hline Baseline & 3.63 & 0.49 & $3 \cdot 50$ & 0.51 & 3.47 & 0.51 & 0.4 \\
\hline 1-month & 1.57 & 0.50 & 1.40 & 0.50 & 1.80 & 0.48 & 0.009 \\
\hline 6-months & 1.50 & 0.51 & 1.40 & 0.50 & 1.73 & 0.45 & 0.028 \\
\hline \multicolumn{8}{|c|}{ Moisture \% } \\
\hline Baseline & $92.59 \%$ & $4.61 \%$ & $91.58 \%$ & $5.28 \%$ & $91.15 \%$ & $4.96 \%$ & 0.514 \\
\hline 1-month & $49 \cdot 34 \%$ & $5.88 \%$ & $48.93 \%$ & $6.62 \%$ & $55.89 \%$ & $9.20 \%$ & $<0.001$ \\
\hline 6-months & $49.42 \%$ & $6.32 \%$ & $48.90 \%$ & $6.79 \%$ & $56.21 \%$ & $7 \cdot 98 \%$ & $<0.001$ \\
\hline \multicolumn{8}{|l|}{ PSS } \\
\hline 1-month & $3 \cdot 53$ & 0.57 & $3 \cdot 77$ & 0.43 & 3.03 & 0.56 & $<0.001$ \\
\hline 6-months & $3 \cdot 37$ & 0.67 & $3 \cdot 73$ & 0.52 & 2.47 & 0.51 & $<0.001$ \\
\hline \multicolumn{8}{|l|}{ GAIS } \\
\hline 1-month & $3 \cdot 37$ & 0.56 & $3 \cdot 77$ & 0.43 & 3.03 & 0.56 & $<0.001$ \\
\hline 6-months & $3 \cdot 37$ & 0.67 & 3.83 & 0.38 & 1.83 & 0.79 & $<0.001$ \\
\hline \multicolumn{8}{|l|}{ DLQI } \\
\hline 1-month & 24.00 & 1.72 & 25.23 & 2.03 & 22.40 & 1.16 & $<0.001$ \\
\hline 6-months & 24.13 & 2.01 & 25.60 & 2.22 & 22.30 & 0.65 & $<0.001$ \\
\hline
\end{tabular}

\section{Hyperhidrosis Disease Severity Score}

At baseline, the average mean \pm SD of HDSS of the laser 980 group was $3.70 \pm 0.47$, the laser 1470 group was $3.57 \pm 0.50$ and the BTA group was $3.63 \pm 0.49(\mathrm{p}=0.572)$.

After the treatment (1-month), mean HDSS scores of the three groups revealed remarkably better reduction from the baseline $(\mathrm{p}<0.001)$. Comparing the three groups at 1 month, post-hoc tests revealed that the laser 980 group and the laser 1470 group had significantly higher reductions of mean HDSS scores than the BTA group with $1.37 \pm 0.49$ and $1.20 \pm 0.41$ versus $2.03 \pm 0.67$, respectively (p-values $<0.001$ ). However, both types of lasers were comparable $(\mathrm{p}=0.688)$.

The same was encountered at the 6-month evaluation where mean HDSS scores of the three groups revealed remarkably better reduction from the baseline $(p<0.001)$. Comparing between the three groups at 6 months, post-hoc tests revealed that the laser 980 group and the laser 1470 group had a significantly higher reduction of mean HDSS score than the BTA group with $1.37 \pm 0.48$ and $1.20 \pm 0.41$ versus $2.20 \pm 0.55$, respectively (p-values $<0.001$ ). However, both types of lasers were comparable $(\mathrm{p}=0.863)$.

\section{Moisture percent}

At baseline, the average mean \pm SD of moisture percentage of the laser 980 group was $92.59 \pm 4.61 \%$, the laser 1470 group was $91.58 \pm 5.28 \%$ and the BTA group was $91.15 \pm 4.96 \%$ (p $=0.514)$.

After the treatment (1-month), mean moisture percentages of the three groups revealed remarkably better reduction from the baseline $(\mathrm{p}<0.001)$. Comparing the three groups at 1 month, post-hoc tests revealed that the laser 980 group and the laser 1470 group had a significantly higher reduction of mean 
moisture percentages than the BTA group with $49.34 \pm 5.88 \%$ and $48.93 \pm 6.62 \%$ versus $55.89 \pm 9.20 \%$, respectively (p-values $0.003 \& 0.001)$. However, both types of lasers were comparable $(\mathrm{p}=1.0)$.

The same was encountered at the 6-month evaluation where mean moisture percentages of the three groups revealed remarkably better reduction from the baseline $(p<0.001)$. Comparing the three groups at 6 months, post-hoc tests revealed that the laser 980 group and the laser 1470 group had significantly higher reductions of the mean moisture percentages than the BTA group with $49.42 \pm 6.32 \%$ and $48.90 \pm 6.79 \%$ versus $56.21 \pm 7.98 \%$, respectively (p-values $0.001 \&<0.001)$. However, both types of lasers were comparable $(\mathrm{p}=1.0)$.

\section{Malodour Score}

At baseline, the average mean $\pm \mathrm{SD}$ of Malodour score of the laser 980 group was $3.63 \pm 0.49$, the laser 1470 group was $3.50 \pm 0.51$ and the BTA group was $3.47 \pm 0.51(\mathrm{p}=0.4)$. After the treatment (1-month), mean Malodour scores of the three groups revealed remarkably better reduction from the baseline $(\mathrm{p}<0.001)$. Comparing the three groups at 1 month, post-hoc tests revealed that the laser 1470 group had significantly higher reductions of mean Malodour scores than the BTA group with $1.40 \pm 0.50$ versus $1.80 \pm 0.48$, respectively ( $p$ $=0.007)$. However, both types of lasers were comparable ( $\mathrm{p}$ $=0.589)$. In addition, laser 980 and BTA were comparable $(\mathrm{p}=0.215)$.

The same was encountered at 6-month evaluation where mean Malodour scores of the three groups revealed remarkably better reduction from the baseline $(p<0.001)$. Comparing between the three groups at 6 month, post-hoc tests revealed that the laser 1470 group had significantly higher reduction of mean Malodour score than the BTA group with $1.40 \pm 0.50$ versus $1.73 \pm 0.45$, respectively $(p=0.028)$. However, both types of lasers were comparable $(p=1.0)$. Also, laser 980 and BTA were comparable $(\mathrm{p}=0.199)$.

\section{Patient Satisfaction Score (PSS)}

Comparing the three groups at the1-month visit, there were significant $(p<0.001)$ differences between groups with regards to PSS. It was $3.5 \pm 0.57$ in the laser 980 group, $3.77 \pm 0.43$ in the laser 1470 group and $3.03 \pm 0.56$ in the BTA group. Comparing the three groups, post-hoc tests revealed that the laser 980 and the laser 1470 groups had significantly higher PSS than the BTA group (p-values $0.001 \&<0.001$, respectively). However, both types of lasers were comparable $(\mathrm{p}=0.263)$.

In addition, comparing between the three groups at the 6 -month visit, there were significant $(p<0.001)$ differences between groups with regards to PSS. It was $3.37 \pm 0.67$ in the laser 980 group, $3.73 \pm 0.52$ in the laser 1470 group and
$2.47 \pm 0.51$ in the BTA group. Comparing the three groups, post-hoc tests revealed that the laser 980 and the laser 1470 groups had significantly higher PSS than the BTA group (pvalues $<0.001 \&<0.001$, respectively). In addition, laser 1470 has higher PSS than laser $980(\mathrm{p}=0.044)$.

\section{Global Aesthetic Improvement Scale (GAIS)}

Comparing between the three groups at 1-month visit, there was a significant $(p<0.001)$ differences between groups with regards GAIS. It was $3.37 \pm 0.56$ in the laser 980 group, $3.77 \pm 0.43$ in the laser 1470 group and $3.03 \pm 0.56$ in the BTA group. Comparing between the three groups, post-hoc tests revealed that the laser 980 and the laser 1470 groups had significantly higher GAIS than the BTA group (p-values 0.043 $\&<0.001$, respectively). Also, laser 1470 has higher GAIS than laser $980(\mathrm{p}=0.011)$.

In addition, comparing between the three groups at 6-month visit, there was a significant $(\mathrm{p}<0.001)$ differences between groups with regards GAIS. It was $3.37 \pm 0.67$ in the laser 980 group, $3.83 \pm 0.382$ in the laser 1470 group and $1.83 \pm 0.79$ in the BTA group. Comparing between the three groups, posthoc tests revealed that the laser 980 and the laser 1470 groups had significantly higher GAIS than the BTA group (p-values $<0.001 \&<0.001$, respectively). In addition, laser 1470 has higher GAIS than laser $980(\mathrm{p}=0.017)$.

\section{Quality of life by Dermatology's Living Quality of Life Index}

The laser 980 and the laser 1470 groups demonstrated with significantly better improvement for their quality of life by DLQI score than the BTA group at both 1-month visit (pvalue $=0.010)$ and 6 -months visit $(p$-values $<0.001)$ (Table $1 \&$ Figure 2). Also, post-hoc tests showed that the laser 1470 showed higher DLQI scores at 1-month and 6-months visits than the laser 980 (p-values $0.016 \& 0.006$ ).

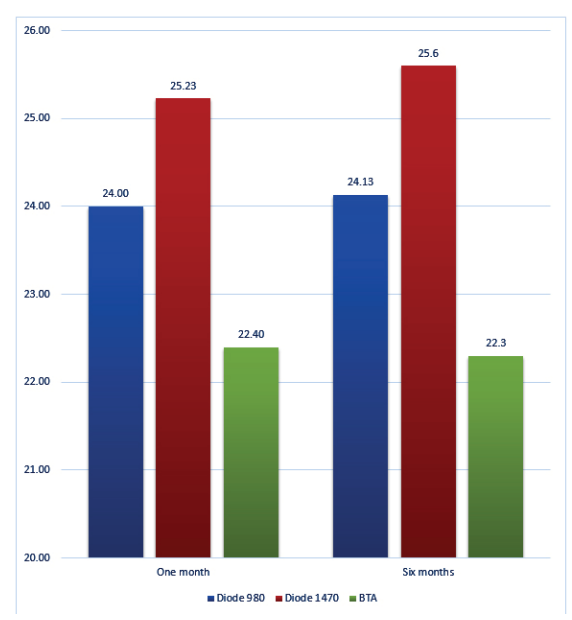

Figure 2: Quality of life by Dermatology's Living Quality of Life Index. 


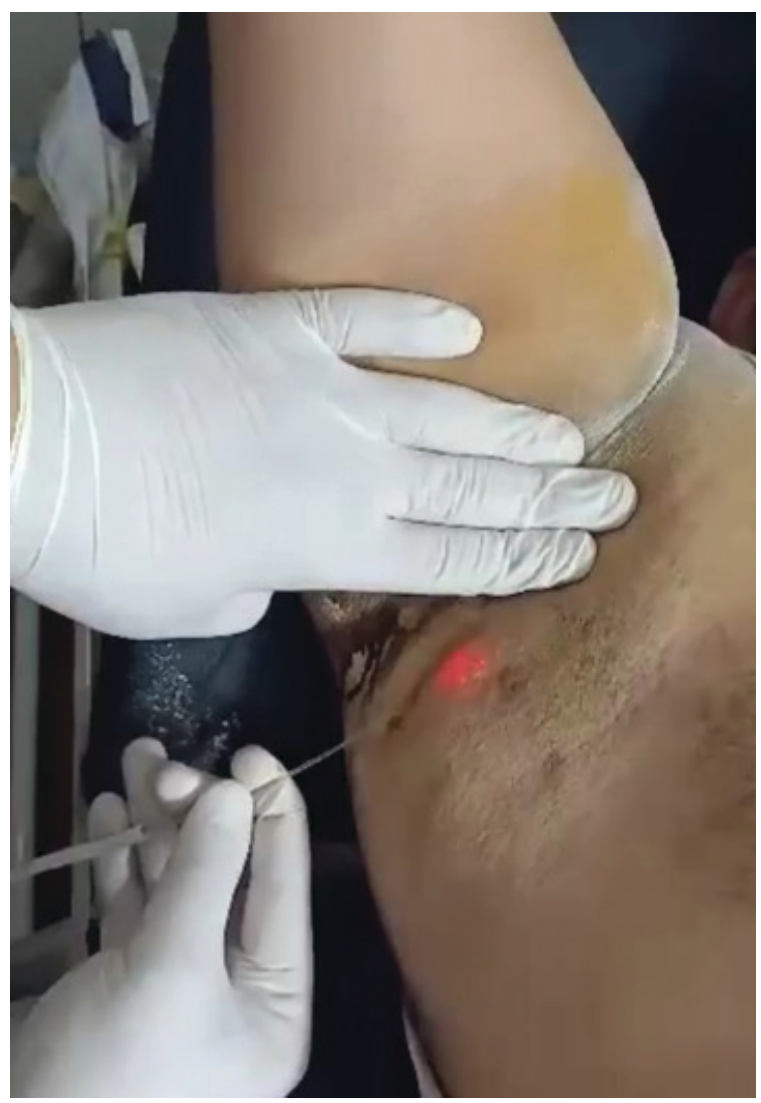

Figure 3: Diode Laser $1470 \mathrm{~nm}$ for axillary hyperhidrosis.

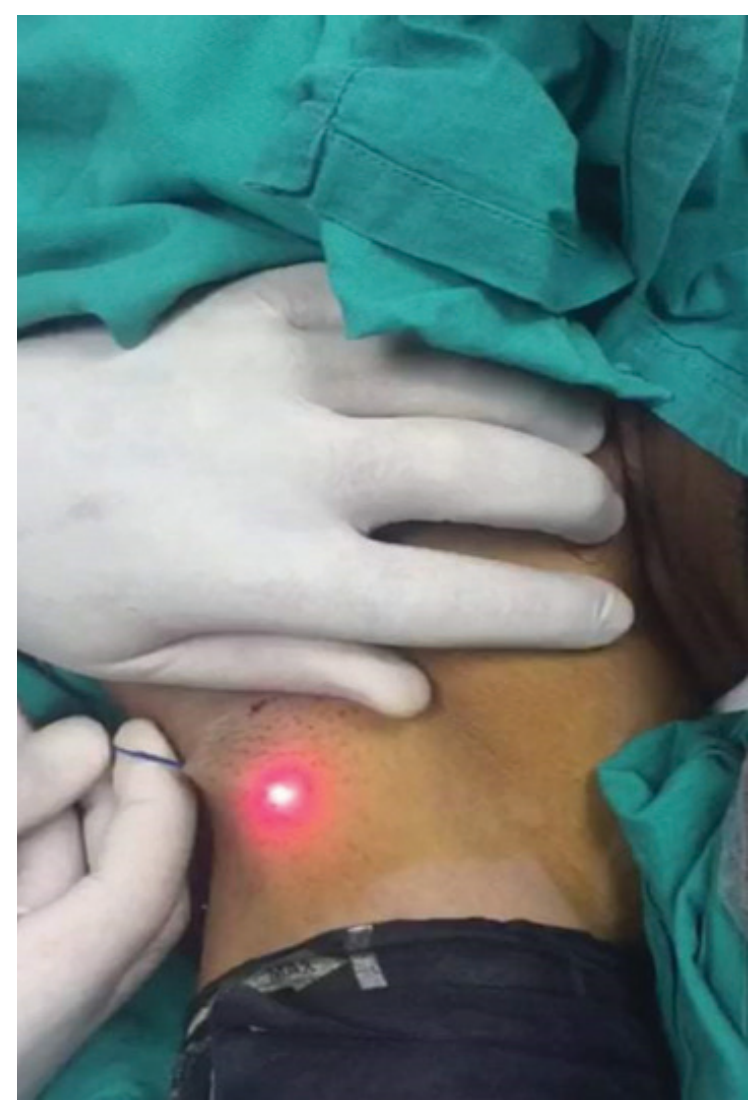

Figure 4: Diode Laser 980 nm for axillary hyperhidrosis.

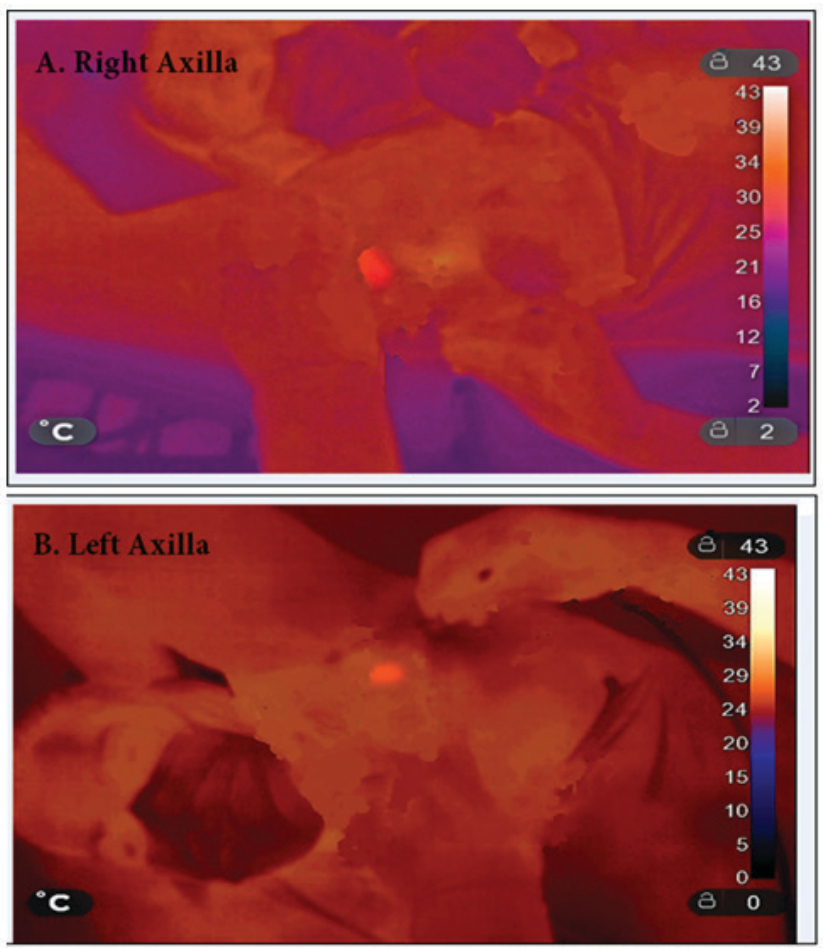

Figure 5: (5.A) Thermal camera of Right Axilla (5.B) Thermal camera of Left Axilla during lasing procedure for axillary hyperhidrosis (According to the thermal camera readings, the temperature did not exceed 40-45 degree Celsius).

\section{Adverse effects}

Decreased hair growth was seen in 15, 25, 3 cases in the laser 980, the laser $1470 \&$ BTA groups, respectively $(\mathrm{p}<0.001)$. In addition, Skin color changes were seen in 6, 20, 2 cases in the laser 980, the laser 1470 \& BTA groups, respectively $(\mathrm{p}<0.001)$.

\section{DISCUSSION}

The current study enrolled ninety PAH cases randomized into three groups; the laser 980 group, the laser 1470 group \& the BTA group. After one month and 6 months of treatment, the mean HDSS scores and the mean moisture percentages of the three groups revealed remarkably better reduction from the baseline. The laser 980 group and the laser 1470 group had significantly higher reduction of mean HDSS score than the BTA group. However, both types of lasers were comparable. The mean Malodour scores of the three groups revealed remarkably better reduction from the baseline after one month and six months. The laser 1470 group had significantly higher reduction of mean Malodour score than the BTA group. Both types of lasers as well as laser 980 and BTA were comparable.

Comparing the three groups at the 1-month visit, there was a significant difference between groups with regards to PSS. 
The laser 980 and the laser 1470 groups had significantly higher PSS than the BTA. However, both types of lasers were comparable. However, at month six, the same results were encountered apart from that laser 1470 has higher PSS than laser 980. At 1-month and 6-month visits, there were significant differences between groups with regards GAIS score. The laser 980 and the laser 1470 groups had significantly higher GAIS than the BTA group. In addition, laser 1470 has higher GAIS than laser 980. The laser 980 and the laser 1470 groups demonstrated with significantly better improvement for their quality of life by DLQI score than the BTA group at both 1-month visit and 6-months visit. Posthoc tests showed that the laser 1470 showed higher DLQI scores at 1-month and 6-months visits than the laser 980.

About $1-3 \%$ of the general population suffers from $\mathrm{PAH}$, which has a direct impact on their quality of life. ${ }^{27,28}$ Hyperhidrosis, the excessive sweating above what is biologically necessary for the body to maintain its temperature, can affect a person's social life, mental health, and capacity to work or study. ${ }^{1}$

Hyperhidrosis has been associated to a considerable decrease in health-related quality of life, especially during the summer months. Job and social connections restrictions, physical and recreational activity restrictions, and emotional and mental health concerns are all examples. ${ }^{29}$

In research, hyperhidrosis has been related to higher levels of anxiety and melancholy. Excessive sweating may be embarrassing and might interfere with daily activities at school, work, and other social contexts. Sweaty people are always worried about how much they sweat and spend hours each day dealing with it.$^{30}$ To the best of the available literature, no direct comparison between diode laser $980 \mathrm{~nm}$, diode laser $1470 \mathrm{~nm}$ and BTA in the treatment of PAH could be found.

Cervantes et al. (2018) did a comprehensive assessment of the literature on the use of lasers in the management of hyperhidrosis, focusing on the Neodymium: Yttrium-Aluminum-Garnet (Nd: YAG) and diode lasers. On March 27, 2017, the query word "hyperhidrosis AND laser" was used to search the PubMed database of the National Library of Medicine. This search yielded 90 results. Two reviewers independently assessed the studies' eligibility and evaluated their methodological quality. Publications not relevant to the use of a laser modality for the treatment of axillary hyperhidrosis were eliminated. Six articles were eventually included as a consequence of this process. ${ }^{31}$

The review showed that there are few studies on the use of laser treatment for hyperhidrosis in the literature. The studies looked at a total of 160 individuals who had PAH and were treated with laser treatment. A total of 39 patients were treated with the Nd: YAG laser, according to four studies. A total of 121 patients were treated with diode laser in two articles. Cervantes et al. (2018) concluded that because of its known efficacy and low side effect profile, the Nd: YAG laser may be a possible therapeutic option for hyperhidrosis, according to our findings. However, further large, randomized controlled trials are required to prove the treatment option's safety and efficacy. ${ }^{31}$

The use of Nd: YAG for the treatment of PAH in 31 women and eight men, ranging in age from 18 to 55 years, was recorded in two prospective investigations, one case report, and one randomized control traial. ${ }^{15}$ The laser parameters differed from study to study. ${ }^{11,13,14, \text { and } 15}$

In 2008, Goldman and Wollina conducted a pilot research in 17 patients using the $1064 \mathrm{~nm} \mathrm{Nd:} \mathrm{YAG} \mathrm{laser} \mathrm{to} \mathrm{treat} \mathrm{PAH.}$ Patients were monitored for 12- 43 months after receiving one treatment in each axilla. The results were encouraging, with the Physician's Global Assessment rating 82.3 percent of the results as good or better, indicating considerable clinical improvement. ${ }^{11}$

The use of the $1064 \mathrm{~nm}$ for PAH was also supported by Minor's starch-iodine test and the patient's self-assessment. After laser therapy, histologic analysis revealed significant changes in the eccrine glands, ranging from microvesiculation and decapitation to full vaporization. At 5 months after treatment, only one of the 17 treated patients relapsed and required another laser treatment. One patient experienced burns, another experienced seroma development, and eight patients experienced transient hair loss. ${ }^{11}$

Three years later, Kotlus described a 39-year-old African American lady with treatment-resistant axillary hyperhidrosis. Subdermal $1320 \mathrm{~nm}$ Nd: YAG laser therapy was conducted after numerous failed efforts with topical aluminum salts, BTA injections, and thoracic sympathectomy. Complete remission was sustained 18 months after therapy, with no indication of compensatory sweating or side consequences..$^{13}$

Six patients with axillary hyperhidrosis were treated with monthly $1064 \mathrm{~nm} \mathrm{Nd:} \mathrm{YAG} \mathrm{laser} \mathrm{sessions} \mathrm{at} \mathrm{laser} \mathrm{hair} \mathrm{re-}$ moval settings on one axilla, while the opposite axilla served as a control in a prospective, randomized, case-controlled pilot research by Letada et al. ${ }^{14}$ In comparison to the control group, investigators found a statistically significant improvement in subjective sweating ratings after 5-6 sessions. In addition, 30 days after treatment, all participants had significantly less sweating on the treated axilla compared to the control axilla, as measured by the modified starch-iodine test. The authors found that long-pulse Nd:YAG laser hair removal can enhance sweat output subjectively and objectively for at least 9 months after treatment beginning in typical circumstances. In terms of sweat gland density, staining features, and overall sweat gland morphology, histologic examination of axillary tissue revealed no significant differences between pre- and post-treatment. ${ }^{14}$ 
The use of a 1440-nm Nd: YAG laser for the treatment of focal PAH was studied by Caplin and Austin. The eccrine gland is mostly made up of water, thus the $1440 \mathrm{~nm}$ wavelength was thought to be particularly helpful in treating PAH due to its increased affinity for water absorption. Following a one-year follow-up, all patients reported clinical and quantitative improvements. ${ }^{15}$

This study, demonstrated the efficacy of eccrine gland necrosis therapy. The Nd: YAG laser's mode of action for the repression/elimination of hyperhidrosis was revealed by histological examination of treatment biopsy samples, which revealed apocrine gland necrosis. Temporary numbness in treated patients, discomfort in 14 patients, swelling in patients diagnosed, bruising in 13 cases, and itching in 11 treated patients were among the adverse effects. ${ }^{15}$

Diode lasers are solid-state laser systems that are commonly used for hair removal and are gaining popularity due to their great penetration depth and capacity to handle people with darker skin..$^{32}$ They have lately been approved for the treatment of hyperhidrosis. In a total of 131 patients, two randomized controlled studies demonstrated the utility of a diode laser for the treatment of PAH. ${ }^{31,32}$

Because it penetrates the dermis to target melanin, the 800 $\mathrm{nm}$ pulsed diode laser is widely used for hair removal. Bechara et al. examined the impact of a long-pulsed $800 \mathrm{~nm}$ diode laser on the sweat rate of individuals with PAH after many reports of reduced sweating following axillary laser hair eradication. The results of a 19-patient half-side controlled study revealed a statistically significant decrease in perspiration rate on the treated side following laser treatment. Surprisingly, the untreated group showed a smaller but statistically significant decrease in sweat rate, suggesting the possibility of a psychological (placebo) impact. The lack of a statistically significant difference between the treated and untreated groups, as well as no significant damage or change to the eccrine or apocrine sweat glands on histological examination, led researchers to conclude that, while laser epilation may reduce sweat rates, the placebo effect is more likely to be to bear responsibility than a direct therapeutic effect of laser epilation. The sole side effect recorded was axillary skin depigmentation in one patient, which resolved with time. Perhaps the low dose of laser is behind that result. ${ }^{33}$

Leclere et al. investigated the effectiveness of four different surgical techniques on 100 cases with PAH in a comparativecontrastive research. Patients were given one of 4 treatment options: (1) $975 \mathrm{~nm}$ laser, (2) 924/975 nm laser, (3) cannula curettage, or (4) laser 924/975 nm followed by cannula curettage. ${ }^{34}$

Subjective criteria (patient reported compensatory sweating and patient subjective efficacy satisfaction via GAIS) and objective criteria (HDSS and starch-iodine test) showed that the combination of laser 924 and $975 \mathrm{~nm}$ and curettage (group 4) was the best treatment option for PAH after a oneyear follow-up. Laser $975 \mathrm{~nm}$ monotherapy proved ineffective for PAH, causing severe burns and lasting scarring in two patients. ${ }^{34}$

Although the outcomes of the 924/975 nm laser (group 2) and curettage (group 3) were similar, traditional curettage was the superior option of the two. The researchers found that 924/975 nm laser therapy coupled with curettage (group 4) was a safe treatment with long-lasting effects after a year, owing to the extra damage produced by curettage to tissues only partially destroyed by laser activity. The only adverse events reported in group 4 were bruising in one patient and transient loss of feeling in another. However, nothing was reported about the baseline characteristics and demographic data of the four groups. Moreover, the randomization technique was not mentioned and the number of patients lost up the follow-up. ${ }^{34}$

Laser light is used to permanently destroy the axillary sweat glands by photo selectively heating sweat gland tissue without damaging any of the surrounding tissue. Some researchers found that Nd: YAG $1064 \mathrm{~nm}$ laser can be successfully used in axillary hyperhidrosis to cause controlled destruction of eccrine and apocrine units. ${ }^{14}$

On the other hand, Neumann et al. (2013) conducted an evidence-based evaluation that found BTA injection to be effective in the treatment of PAH in 923 individuals. ${ }^{35}$ Heckmann et al. (2001) conducted a randomized controlled trial to test 200 units of BTA to a placebo with a 26-week follow-up period and found that sweat production was reduced by gravimetric measurement. ${ }^{6}$ Neumann et al. (2001) included 320 patients with $\mathrm{PAH}$ in a 16 -week trial to compare 50 units of BTA to a placebo, and BTA demonstrated considerably superior sweat gland output decrease by gravimetric assessment than the placebo. ${ }^{7}$

Dry eyes and skin were two of the most prevalent adverse effects of BTA. However, in our research, we were unable to identify such impact. Lowe et al. (2007) performed a trial with $322 \mathrm{PAH}$ patients to compare three arms: 50 units of A/Ona per axilla, 75 units of A/Abo per axilla, and placebo, with a one-year follow-up. ${ }^{8}$

Both botulinum toxin groups showed a percent change in at least two levels of HDSS in this research, which was substantially better (75\%) than the placebo (25\%) (p 0.001). The therapeutic impact of botulinum toxin lasted longer than the placebo. ${ }^{8}$

The most frequent approach for temporarily disabling sweat glands is to employ botulinum toxin. It is quite safe, but the main problem is that the treatment is not permanent, and the control lasts on average just six months. After six months, the patient will require further injections. It becomes extremely 
costly in the long run. ${ }^{36}$ Only a BTA is now FDA-approved for the treatment of hyperhidrosis by the US Food and Drug Administration. Patients who do not react to BTA treatment may benefit from BTB. ${ }^{37}$

One advantage of our current research is that it is the first randomized control trial compared the three management options for (PAH) with enough sample size. Another advantage is that it included both the subjective and the objective assessment score for efficacy and quality of life. However, one limitation of our study is that it lacked long-term (1year) follow-up of patients.

\section{CONCLUSION}

In conclusion, the current study showed that Diode laser $1470 \mathrm{~nm}$ is a safe and an effective treatment for PAH more than Diode laser $980 \mathrm{~nm}$ that may be attributed to its less penetration skin depth and its high affinity to water.

\section{ACKNOWLEDGMENT}

Authors acknowledge the immense help received from the scholars whose articles are cited and included in references of this manuscript. The authors are also grateful to authors / editors / publishers of all those articles, journals and books from where the literature for this article has been reviewed and discussed.

Source of Funding: The study was an investigator-initiated study that was self-funded only by the principal investigator. No fund was gained for this work.

Ethical approval: This study was approved by the Ethics Committee of the National Institute of Laser in Cairo University, Cairo, Egypt. Clearance letter number \#: 020-001

Conflict of interest: The authors declare no competing interests.

\section{Authors' Contribution}

\begin{tabular}{|l|l|}
\hline Author name & Contribution \\
\hline 1) Diaa Aldin Sayed Ibrahim & $\begin{array}{l}\text { Conceptualizing the idea of } \\
\text { the study, study design, con- } \\
\text { ducting the study, statistical } \\
\text { analysis, writing the paper, } \\
\text { and revision of the paper. }\end{array}$ \\
\hline 2) Mahmoud S. Elbasiouny & $\begin{array}{l}\text { Conceptualizing the idea of } \\
\text { the study and study design. }\end{array}$ \\
\hline 3) Tarek F. Elwakil & $\begin{array}{l}\text { Conceptualizing the idea of } \\
\text { the study and study design. }\end{array}$ \\
\hline 4) Nevien Ahmed Samy & $\begin{array}{l}\text { Statistical analysis and revi- } \\
\text { sion of the paper. }\end{array}$ \\
\hline 5) Maha Rafie Abo Eitta & Revision of the paper. \\
\hline
\end{tabular}

\section{REFERENCES}

1. McConaghy JR, Fosselman D. Hyperhidrosis: management options. Am Fam Physician. 2018; 97(11): 729-734.

2. Cohen JL, Cohen G, Solish N, Murray CA. Diagnosis, impact, and management of focal hyperhidrosis: treatment review including botulinum toxin therapy. Facial Plast Surg Clin North Am. 2007; 15:17-30 v-vi

3. Atkins JL, Butler PE. Hyperhidrosis: a review of current management. Plast Reconstr Surg. 2002; 110:222-228.

4. Nawrocki S, Cha J. The etiology, diagnosis, and management of hyperhidrosis: A comprehensive review: Therapeutic options. J Am Acad Dermato. 2019; 181 (3):669-680. https:// doi. org/ 10. 1016/j. jaad. 2018. 11. 066

5. Rummaneethorn P, Chalermchai T. A comparative study between intradermal botulinum toxin A and fractional microneedle radiofrequency (FMR) for the treatment of primary axillary hyperhidrosis. Lasers Med Sci. 2020 Jul; 35(5):1179-1184.

6. Heckmann M, Ceballos-Baumann AO, Plewig G, Hyperhidrosis Study G. Botulinum toxin A for axillary hyperhidrosis (excessive sweating). N Engl J Med. 2001; 344:488-493.

7. NaumannM, Lowe NJ. Botulinum toxin type A in treatment of bilateral primary axillary hyperhidrosis: randomised, parallel group, double blind, placebo controlled trial. BMJ. 2001; 323:596-599.

8. Lowe NJ, Glaser DA, Eadie N, Daggett S, Kowalski JW, Lai PY, et al. Botulinum toxin type $\mathrm{A}$ in the treatment of primary axillary hyperhidrosis: a 52-week multicenter double-blind, randomized, placebo-controlled study of efficacy and safety. J Am Acad Dermatol. 2007 Apr;56(4):604-11. doi: 10.1016/j.jaad.2007.01.009. Epub 2007 Feb 15. PMID: 17306417.

9. Solish N, Benohanian A, Kowalski JW; Canadian Dermatology Study Group on Health-Related Quality of Life in Primary Axillary Hyperhidrosis. Prospective open-label study of botulinum toxin type A in patients with axillary hyperhidrosis: effects on functional impairment and quality of life. Dermatol Surg. 2005 Apr;31(4):405-13. PMID: 15871315.

10. Ashley L. Brown, Jennifer G, and Samantha H. Hyperhidrosis: review of recent advances and new therapeutic options for primary hyperhidrosis. Curr Opin Pediatr 2014, 26:460-465.

11. Goldman A., Wollina U. Subdermal Nd-YAG laser for axillary hyperhidrosis. Dermatol Surg. 2008;34: 756 - 762.

12. Ichikawa K, Miyasaka M, Aikawa Y. Subcutaneous laser treatment of axillary osmidrosis: a new technique. Plast Reconstr Surg. 2006;118(1): 170-174.

13. Kotlus BS. Treatment of refractory axillary hyperhidrosis with a 1320-nm Nd: YAG laser. J Cosmet Laser Ther. 2011;13(4):193195.

14. Letada PR, Landers JT, Uebelhoer NS, Shumaker PR. Treatment of focal axillary hyperhidrosis using a long-pulsed $\mathrm{Nd}$ : YAG $1064 \mathrm{~nm}$ laser at hair reduction settings. J Drugs Dermatol. 2012;11(1):59-63.

15. Caplin D, Austin J. Clinical evaluation and quantitative analysis of axillary hyperhidrosis treated with a unique targeted laser energy delivery method with 1-year follow-up. J Drugs Dermatol. 2014;13(4):449-456.

16. Klopper M, Fisher G, Blugerman G. Laser-Assisted Suction of Sweat Glands and Axillary Epilation. Shiffman MA, Di Giuseppe A, editors. Liposuction principles and practice. Berlin: Springer-Verlag Berlin, 2006; 505-15. 2.

17. Schavelzon DE, Blugerman G, Chomyszyn A, Ponton JA, Lanfranchi LA, Martinez LA. Noninvasive treatment of axillary hyperhidrosis with Nd: YAG laser. UpDate in Plastic Surgery, 2010; Vol. 3, No. 1. 
18. Aydin F., Pancar GS., Senturk N., Bek Y., Yuksel EP., Canturk T., et al. Axillary hair removal with 1064-nm Nd: YAG laser increases sweat production. Clin Exp Dermatol. 2010;35: 588 -592 .

19. Mordon SR, Trelles MA, Leclere FM, Betrouni N. New treatment techniques for axillary hyperhidrosis. J Cosmet Laser Ther. 2014 Oct;16(5):230-5. doi: 10.3109/14764172.2014.948883. Epub 2014 Aug 25. PMID: 25065458.

20. Obeid G., Helou J., Maatouk I., Moutran R., Tomb R. Depilatory laser: a potential causative factor for inguinal hyperhidrosis: Report of three cases. J Cosmet Laser Ther. 2013;15: 286 - 289.

21. Mordon S., Plot E. Laser lipolysis versus traditional liposuction for fat removal. Expert Rev Med Devices. 2009; 6: 677 - 688.

22. Maletic D., Maletic A., Vizintin Z. Laser Assisted Reduction of Axillary Hyperhidrosis (LARAH) evaluation of success up to 24 months after the treatment. J Laser Health Academy. 2011; 1: $37-42$.

23. Tarhan IA, Dumantepe M, Yurdakul I, Kehlibar T, Ozler A. Local cooling effect on perforation rates comparing the $980-1470$ $\mathrm{nm}$ laser wavelengths used with endovenous laser ablation: double blind in-vitro experimental study. Phlebology. 2014; 29:120-125.

24. Solish N, Bertucci V, Dansereau A, Hing HC, Lynde C, Lupin M, et al. Hyperhidrosis Disease Severity Scale, A Comprehensive Approach to the Recognition, Diagnosis, and SeverityBased Treatment of Focal Hyperhidrosis: Recommendations of the Canadian Hyperhidrosis Advisory Committee. Dermatol Surg., 2007; 33(8): 908-23.

25. Park YJ, Shin MS. What is the best method for treating osmidrosis? Ann Plast Surg 2001;47:303-9.

26. Jung JM, Na HM, Kim JH, Yoon J, Yang HJ, Lee WJ, et al. The efficacy and safety of a fractional microneedle radiofrequency device for the treatment of axillary hyperhidrosis: clinical prospective pilot study. Lasers Med Sci. 2021 Aug 28. doi: 10.1007/ s10103-021-03404-z. Epub ahead of print. PMID: 34453655.

27. Walling HW, Swick BL. Treatment options for hyperhidrosis. Am J Clin Dermatol. 2011; 12(5):285-295.

28. Doolittle J,Walker P, Mills T, Thurston J. Hyperhidrosis: an update on prevalence and severity in the United States. Arch
Dermatol Res. 2016 308(10):743-749. https://doi.org/10.1007/ s00403-016-1697-9

29. Glaser DA, Hebert A, Pieretti L, Pariser D. Understanding Patient Experience With Hyperhidrosis: A National Survey of 1,985 Patients. J Drugs Dermatol. 2018 Apr 1;17(4):392-396. PMID: 29601615.

30. Kamudoni P., MuellerB, Halford J., Schouveller A., Stacey B., Salek MS.: The impact of hyperhidrosis on patients' daily life and quality of life: a qualitative investigation. Health and Quality of Life Outcomes. 2017;V.15(1) DOI 10.1186/s12955-017.

31. Cervantes J, Perper M, Eber AE, Fertig RM, Tsatalis JP, Nouri $\mathrm{K}$. Laser treatment of primary axillary hyperhidrosis: a review of the literature. Lasers Med Sci. 2018 Apr 1;33(3):675-81.

32. Puri N. Comparative study of diode laser versus neodymiumyttrium aluminum: garnet laser versus intense pulsed light for the treatment of Hirsutism. J Cutan Aesthet Surg. 2015; 8(2):97101. https://doi.org/10.4103/0974-2077.158445.

33. Bechara FG, Georgas D, Sand M, Stucker M, Othlinghaus N, Altmeyer P, et al. Effects of a long-pulsed 800-nm diode laser on axillary hyperhidrosis: a randomized controlled half-side comparison study. Dermatol Surg. 2016; 38(5):736-740. https://doi. org/10.1111/j.1524-4725.2012.02339.x

34. Leclere FM, Moreno-Moraga J, Alcolea JM, Vogt PM, Royo J, Cornejo P, et al. Efficacy and safety of laser therapy on axillary hyperhidrosis after one year follow-up: a randomized blinded controlled trial. Lasers Surg Med. 2015; 47(2):173-179. https:// doi.org/10.1002/lsm.2232433.

35. Naumann M, Dressler D, Hallett M, Jankovic J, Schiavo G, Segal KR, et al. Evidence-based review and assessment of botulinum neurotoxin for the treatment of secretory disorders. Toxicon. 2013 Jun 1;67:141-52. doi: 10.1016/j.toxicon.2012.10.020. Epub 2012 Nov 23. PMID: 23178324.

36. Hamm H. Defining and Diagnosing Primary and Secondary Hyperhidrosis. European Dermatology, Touch Briefings 2009: 82-4.

37. Farrugia MK, Nicholls EA. Intradermal botulinum A toxin injection for axillary hyperhidrosis. J Pediatr Surg 2005;40:16681669. 